\section{ADTH-02 TRANS-ANAL SUBMUCOSAL ENDOSCOPIC RESECTION (TASER) FOR COMPLEX RECTAL POLYPS}

${ }^{1}$ Ioannis Stasinos*, ${ }^{2}$ ZP Tsiamoulos, 'Vasilios Papastergiou, 'Rajaratnam Rameshshanker, ${ }^{1}$ Adel Polecina, ${ }^{2}$ Joseph Sebastian, ${ }^{1}$ Aurelia Wawszczak, ${ }^{1}$ Omar Faiz, ${ }^{1} J a n i n d r a$ Warusavitarne, 'BP Saunders. 'St Mark's Hospital \& Academic Institute, London, UK; ${ }^{2}$ QEQM Hospital, East Kent Hospitals University, Kent, UK

\subsection{6/gutjnl-2018-BSGAbstracts. 14}

Introduction Trans-Anal Submucosal Endoscopic Resection (TASER) is a novel instrumental platform for resection of large $(\geq 5 \mathrm{~cm})$ and/or complex rectal polyps (CRPs). It involves a multi-port trans-anal access, enabling dynamic tissue retraction to assist endoscopic submucosal dissection (ESD), used alone or in conjunction with supplementary techniques [Endoscopic Mucosal Resection (EMR) or Ablation (EMA), Trans-Anal Excision (TAE)].

Methods A prospective database (Jan 2013-October 2017) was analysed including consecutive patients undergoing TASER for CRPs in two UK centres.

Results Sixty-four CRPs (median size $7.5 \mathrm{~cm}$; range: 5-18) were resected in 64 patients (36 males, mean 67.1 years): 33 by $\mathrm{ESD}$ and 31 by hybrid procedures $(\mathrm{ESD}+\mathrm{EMR}=14$, ESD $+\mathrm{EMR}+\mathrm{EMA}=6, \quad \mathrm{ESD}+\mathrm{TAE}=4, \quad \mathrm{ESD}+\mathrm{EMR}+\mathrm{TAE}=3, \quad \mathrm{ESD}$ $+\mathrm{EMR}+\mathrm{EMA}+\mathrm{TAE}=4)$. Forty-seven $(73.4 \%)$ lesions were granular-type laterally spreading tumors, 9 (14.1\%) were recurrences, $13(20.3 \%)$ had severe submucosal fibrosis, and 7 $(10.9 \%)$ involved the dentate line. Histology showed lowgrade adenoma/dysplasia in 35 (54.7\%), high-grade adenoma/ dysplasia in $21(32.8 \%), \mathrm{T} 1<1000 \mu \mathrm{m}$ in $5(7.8 \%)$, and $\mathrm{T} 1 \geq 1000 \mu \mathrm{m}$ in $4(6.2 \%)$. In 10 cases a full-thickness dissection was required due to severe submucosal fibrosis $(n=8)$ or T1 cancer $(n=2)$; the defect was closed in $6 / 10$, by using endoclips $(n=2)$ or surgical sutures/endoclips $(n=4)$. All patients were admitted for overnight observation and intravenous antibiotic cover; the median hospital stay was 1 day (range 1-6).

Complete endoscopic excision was achieved in a single session in $60(93.8 \%)$, and 2 sessions in $4(6.2 \%)$ cases. Thirtyeight $(59.4 \%)$ resections were en-bloc, and $36(56.3 \%)$ were histologically complete. Delayed bleeding and post-polypectomy syndrome occurred in $3(4.7 \%)$ and 7 (10.9\%) respectively, with no surgical intervention required. One patient underwent a defunctioning ileostomy due to intra-peritoneal perforation. Of the 9 malignant cases: 4 with $\mathrm{T} 1 \geq 1000 \mu \mathrm{m}$ cancer (2/4 full thickness) were subject to radical surgery, and 5 with $\mathrm{T} 1<1000 \mu \mathrm{m}$ underwent endoscopic surveillance. Endoscopic surveillance data were available for $44(68.8 \%)$ patients, with a median (range) follow-up of 9.2 (3-43.5) months. The local recurrence was $13.6 \%$; in all cases treated endoscopically. To date, the overall rate of patients spared from radical surgery is $60 / 64$ (93.7\%).

Conclusions The current study demonstrates favorable clinical outcomes of TASER, offering surgery-sparing cure in $>90 \%$ of patients with CRPs. The use of a transanal platform to allow a flexible resection approach, enabling both endoscopic and trans-anal resection techniques, appears to be advantageous.

\section{ADTH-03 MISOPROSTOL FOR THE HEALING OF ASPIRIN AND NSAID-RELATED SMALL BOWEL ULCERS IN OBSCURE GASTRO-INTESTINAL BLEEDING}

${ }^{1,2}$ Ali Taha*, ${ }^{1}$ Caroline McCloskey, ${ }^{3}$ Paula McSkimming, ${ }^{1}$ Theresa Craigen, ${ }^{1}$ Debbie Callaghan, ${ }^{3}$ Alex McConnachie. ${ }^{1}$ NHS Ayrshire and Arran, Kilmarnock, UK; ${ }^{2}$ University of Glasgow Medical School, Glasgow; ${ }^{3}$ Robertson Centre for BiostatisticsUniversity of Glasgow, Glasgow

\subsection{6/gutjnl-2018-BSGAbstracts. 15}

Introduction The incidence of obscure GI bleeding has been rising. This condition is now accepted to mainly originate from small bowel lesions and to be related to the wide use of low-dose aspirin and NSAIDs. We aimed to assess the efficacy of misoprostol, a prostaglandin analogue, in the healing of small bowel ulcers and erosions in patients using aspirin/ NSAIDs and presenting with obscure GI bleeding (MASTERS Trial).

Methods and design This was a prospective, randomised, placebo-controlled trial, comparing misoprostol $200 \mathrm{ug}$ with placebo taken 4-times daily for 8 weeks, while continuing on low-dose aspirin and/or NSAIDs. Patients were screened if they had one or more of the following: iron deficiency anaemia; a drop in $\mathrm{Hb}$ level of $2.0 \mathrm{gm} / \mathrm{dL}$ or more; and/or positive faecal occult blood test. Those without a potential source of bleeding on their upper GI endoscopy and colonoscopy but then found to have small bowel ulcers and erosions, using video-capsule endoscopy (OMOM Endoscopy System, China), were considered for randomisation. Small bowel lesions were classified as modified from Maiden et al, Clin Gastroenterol Hepatol 2007. Patients were clinically assessed 4 weeks after randomisation and with repeat capsule endoscopy after 8 weeks to check for mucosal healing. Capsule images were examined by two blinded assessors, and any discrepancies were resolved by consensus. The primary endpoint was full healing of small bowel ulcers and/or erosions. Secondary endpoints were changes in the numbers of ulcers and erosions, and haemoglobin $(\mathrm{Hb})$ level.

Results Of 232 patients screened, 102 eligible patients were randomised: 50 took misoprostol and 52 took placebo. Complete healing of ulcers and erosions was observed in 27 (54\%) misoprostol patients and $9(17 \%)$ in the placebo group $(\mathrm{p}<0.001)$. Viewed separately, similar results were seen for healing of ulcers $(68 \%$ vs $17 \%, \mathrm{p}<0.001)$ and erosions $(56 \%$ vs $21 \%, \mathrm{p}<0.001)$. There were no significant changes in $\mathrm{Hb}$ levels over the study period. Compliance did not differ significantly between the two arms $(76 \%$ misoprostol vs $90 \%$ placebo took $>75 \%$ of study drugs, $\mathrm{p}=0.065)$. Mild, moderate, or severe adverse events of interest (abdominal pain, nausea/vomiting, diarrhoea, or other events) were observed in $48 \%$ of misoprostol patients vs $44 \%$ placebo $(p=0.84)$, but tended to be more severe in the misoprostol group $(\mathrm{p}=0.017)$. There were no serious adverse events.

Conclusions Misoprostol is effective in the healing of small bowel ulcers and erosions in patients with obscure gastrointestinal bleeding while using low-dose aspirin and NSAIDs. 